\title{
Chemical Analysis of the Fluorine in Niobium Electropolishing
}

\author{
Denise C. Ford ${ }^{1,2}$, Charlie Cooper ${ }^{1}$, Lance D. Cooley ${ }^{1}$, Chad Thompson ${ }^{1}$, Donald \\ Bouchard $^{3}$, Brian Albee ${ }^{4}$, Sandra Bishnoi ${ }^{4}$ \\ ${ }^{1}$ Fermi National Accelerator Laboratory, Batavia, IL 60510 USA \\ ${ }^{2}$ Northwestern University, Evanston, IL 60208 USA \\ ${ }^{3}$ Anasazi Instruments, Inc., Indianapolis, IN 46203 USA \\ ${ }^{4}$ Illinois Institute of Technology, Chicago, IL 60616 USA
}

email: deniseford@u.northwestern.edu 
Abstract. Surface topography and chemical purity are important factors in niobium superconducting radio-frequency (SRF) cavity performance. Electropolishing (EP) is currently being used to minimize the surface roughness and to remove the damaged surface layer created during cavity manufacturing. This process is not ideal for reasons such as safety and performance consistency, so research and development is ongoing. Furthermore, the EP process specifications have been developed empirically, and a molecular level understanding of the process is not complete. The currently employed polishing solution is composed of sulfuric and hydrofluoric acids, where the fluorine ion is active in polishing the niobium. We used vibrational and nuclear magnetic resonance (NMR) spectroscopies to analyze the standard solution, and show that fluorine is bound and released by the reaction of the acid components in the solution: $\mathrm{HF}+\mathrm{H}_{2} \mathrm{SO}_{4}<->$ $\mathrm{HFSO}_{3}+\mathrm{H}_{2} \mathrm{O}$. This result implies that new recipes can possibly be developed on the principle of controlled release of fluorine by a chemical reaction, which provides a route to improve the safety and effectiveness of EP. We also show that NMR or Raman spectroscopy can be used to monitor the free fluorine when polishing with the standard EP recipe.

Keywords - electropolishing, niobium, NMR, Raman, Infrared, spectroscopy, superconducting radio-frequency cavities 


\section{INTRODUCTION}

The forming and processing of superconducting radio-frequency (SRF) cavities consists of many complex procedures, each of which have the capability to create defects that limit cavity performance. When the required accelerating gradient is greater than 25 $\mathrm{MV} / \mathrm{m}$ electropolishing is used to remove the surface damage created by forming the cavities. The polishing is accomplished with an electric current and viscous electrolyte. The metal part to be polished is placed at the anode of the electrochemical cell, and a counter electrode is placed at the cathode. Negatively charged ions attack and dissolve the metal at the anode, while a viscous solution is used to control the diffusion of these ions and thus the polishing rate.

Electropolishing recipes are usually developed empirically. The standard recipe for niobium [1] contains a 1:9 volume ratio of $49 \%$ hydrofluoric acid, which provides the active polishing ion $\mathrm{F}^{-}$, and $96 \%$ sulfuric acid, which provides a viscous medium to control the polishing rate. Aluminum is used for the cathode. Many studies have been performed by the SRF community to understand the effect of polishing parameters on SRF cavity performance [2-5], as well as the mechanism and quality control of the process [6-13]; however, the detailed chemistry remains elusive. Furthermore, simple inline process monitoring techniques are desired.

The chemistry of niobium electropolishing can be described as a balance between processes that oxidize the metal, processes that dissolve the oxide, and processes that occur in the electropolishing solution. It is characterized by the following reactions [14]: 
electro-oxidation:

$2 \mathrm{Nb}+5 \mathrm{SO}_{4}{ }^{2-}+5 \mathrm{H}_{2} \mathrm{O}->\mathrm{Nb}_{2} \mathrm{O}_{5}+10 \mathrm{H}^{+}+5 \mathrm{SO}_{4}{ }^{2-}+10 \mathrm{e}^{-}$

dissolution:

$\mathrm{Nb}_{2} \mathrm{O}_{5}+6 \mathrm{HF}->\mathrm{H}_{2} \mathrm{NbOF}_{5}+\mathrm{NbO}_{2} \mathrm{~F} \cdot 0.5 \mathrm{H}_{2} \mathrm{O}+1.5 \mathrm{H}_{2} \mathrm{O}$

product formation:

$\mathrm{NbO}_{2} \mathrm{~F} \cdot 0.5 \mathrm{H}_{2} \mathrm{O}+4 \mathrm{HF}->\mathrm{H}_{2} \mathrm{NbOF}_{5}+1.5 \mathrm{H}_{2} \mathrm{O}$

overall:

$2 \mathrm{Nb}+10 \mathrm{HF}+2 \mathrm{H}_{2} \mathrm{O}->2 \mathrm{H}_{2} \mathrm{NbOF}_{5}+5 \mathrm{H}_{2}$

However, what actually occurs during electropolishing is much more complex. During electropolishing, $\mathrm{Nb}_{2} \mathrm{O}_{5}$ is dissolved by fluorine, and the dissolved niobium is kept in solution with a complexing agent. Niobium pentoxide is also dissolved by sulfuric acid but the reactivity is negligible compared to the action of hydrofluoric acid. Sulfuric acid, however, is efficient as a complexing agent. Numerous possible reactions involving the electropolishing solution components and niobium are listed in [15].

In this study, we use vibrational and nuclear magnetic resonance (NMR) spectroscopy to analyze the electropolishing solution. Additionally, we show that NMR or Raman spectroscopy can be used to monitor the free fluorine available for polishing with the standard recipe. 


\section{METHODS}

A Renishaw, inVia, Raman microscope with a $785 \mathrm{~nm}$ diode laser and liquid sampling kit was used to obtain the Raman spectra at the Illinois Institute of Technology (IIT). The fluorescence backgrounds were subsequently removed by manually splining the spectra. A PerkinElmer Spectrum One Infrared spectrometer with a $633 \mathrm{~nm}$ laser and an attenuated total reflectance (ATR) sampling probe was used to obtain the IR spectra at Fermilab, and drifting baselines were also subsequently corrected. Some of the Raman spectra were obtained during a PerkinElmer vendor visit at Fermilab.

The NMR spectra were measured on a $60 \mathrm{MHz}$ NMR instrument at Anasazi Instruments, Inc. (www.aiinmr.com) operating at $56.4 \mathrm{MHz}$ for ${ }^{19} \mathrm{~F}$. Five millimeter (5 $\mathrm{mm}$ ) sample tubes were used with teflon inserts to avoid fluorine reaction with the borosilicate glass. No volume correction was applied to the teflon inserts, but the volume differences were determined to be less than 5\%. A pulse-acquire program was used to acquire the spectrum with 8 scans. The repetition rate was 4 scans per minute to allow

for complete relaxation of the ${ }^{19} \mathrm{~F}$ species for a total measurement time of 2 minutes. Spectra were baseline flattened and integrated with fixed integrated regions using NUTS software (www.acornnmr.com). Chemical shifts are relative to $\mathrm{CFCl}_{3}$ in acetone-d6 solvent. 


\section{RESULTS}

\section{A. Raman}

The spectra for freshly mixed EP solution, 96\% sulfuric acid, and 49\% hydrofluoric acid are shown in Figure 1. Sulfuric acid has several strong peaks in its Raman spectrum while hydrofluoric acid doesn't appear to be distinguishable. Several new peaks $\left(810 \mathrm{~cm}^{-1}\right.$ and $\left.1082 \mathrm{~cm}^{-1}\right)$ appear, however, in the EP solution spectrum that are not present in the sulfuric acid spectrum.

Because sulfuric acid is a very strong acid, $\mathrm{pK}_{\mathrm{a} 1}=-3$ in water, it is plausible that new peaks appear due to deprotonation upon the addition of the hydrofluoric acid solution. To test this hypothesis, we obtained the spectrum of sulfuric acid diluted with water and present the results in Figure 2. The relative intensities of the peaks in the sulfuric acid solution clearly change upon dilution, indicating differing concentrations of the ions comprising the sulfuric acid - water solutions at different concentrations. Namely, the loss of the peak at $1390 \mathrm{~cm}^{-1}$, the change in the ratio between the $\sim 915$, 1050 , and $1150 \mathrm{~cm}^{-1}$ peaks, and the growth of the peak at $\sim 990 \mathrm{~cm}^{-1}$. The peak locations and relative intensities for new the EP and sulfuric acid solutions are listed in Table 1. These spectral changes do not explain the new peaks appearing upon the mixing of sulfuric and hydrofluoric acid, however.

The Raman spectrum of hydrofluoric acid was examined further for subtle features that may be useful for analysis. The spectra at several dilutions are shown in Figure 3. Hydrofluoric acid is a very weak Raman scatterer [16]. Broad weak peaks 
appear around 1800 and $2200 \mathrm{~cm}^{-1}$ for the more concentrated hydrofluoric acid solutions, but they are clearly not suitable for tracking the fluorine concentration in dilute solutions.

Based on the Raman spectra of Gillespie and Robison [17], a potential explanation of the new peaks in the EP solution is the production of fluorosulfonic acid from the reaction of sulfuric and hydrofluoric acids. The reaction is:

$\mathrm{H}_{2} \mathrm{SO}_{4}+\mathrm{HF}<->\mathrm{H}_{2} \mathrm{O}+\mathrm{HFSO}_{3}$

The existence of fluorosulfonic acid in EP solution was previously determined by Eozenou, et al. [11], although they were not able to quantify it by their technique of ion chromatography.

We confirm the existence of fluorosulfonic acid in EP solution by presenting the spectra of EP solution with added fluorosulfonic acid and EP solution with added water, Figure 4. We were not able to obtain spectra of the concentrated fluorosulfonic acid due to safety and handling concerns. The spectrum of EP solution with added water is similar to the dilute sulfuric acid spectrum. Based on these spectra we can attribute the peaks at 1082 and $810 \mathrm{~cm}^{-1}$ to fluorosulfonic acid. Based on the published spectra in [17] we assign the peak at $1082 \mathrm{~cm}^{-1}$ to the $\mathrm{FSO}_{3}{ }^{-1}$ ion, since this peak appears in their spectrum of fluorosulfonic acid with $10 \%$ added water but not in their $100 \%$ fluorosulfonic acid spectrum. Fluorosulfonic acid can be deprotonated in EP solution since it is a stronger acid than both sulfuric and hydrofluoric acids, with a $\mathrm{pK}_{\mathrm{a}}$ of 2.64 in sulfuric acid. 
The Raman spectrum of used EP solution is also shown in Figure 4. The used EP solution spectrum resembles the $96 \%$ sulfuric acid spectrum and the features representative of fluorosulfonic acid are reduced. This indicates that as free $\mathrm{F}^{-}$is used during electropolishing, the fluorosulfonic acid that was produced upon the mixing of the solution components decomposes to produce more hydrofluoric acid, which is in turn used during the EP. Therefore, tracking the strongest fluorosulfonic acid peaks at 1082 and $810 \mathrm{~cm}^{-1}$ can be used as a method to track the amount of fluorine available for EP.

Additional peaks are not evident in the used EP solution spectrum to indicate the formation of any EP products. Based on the experiments of Keller [18] and von Barner et al. [19] niobium fluoride salts should have Raman and IR active vibrations in the 250$1100 \mathrm{~cm}^{-1}$ region. The peaks reported in [18] are listed in Table 2. Two possibilities exist for why these vibrations were not detected in this study: the concentration of the dissolved salts is too low or complexation with sulfuric acid reduces or eliminates their Raman activity.

\section{B. Infrared Spectra}

The infrared spectra of the new EP solution, 96\% sulfuric acid, and $49 \%$ hydrofluoric acid are shown in Figure 5, and their peak locations and relative intensities are listed in Table 3. Several differences exist between the Raman and infrared spectra; notably, hydrofluoric acid has IR active vibrational modes. These modes, however, still do not appear in the EP solution spectrum, as the feature at $\sim 1650 \mathrm{~cm}^{-1}$ is from the water 
(Figure 6). As with Raman spectroscopy, peaks at 808 and $1076 \mathrm{~cm}^{-1}$ appear in the EP solution spectrum and can't be explained by the hydrofluoric acid dilutions (Figure 6) or the sulfuric acid dilutions (Figure 7): these peaks indicate the production of fluorosulfonic acid (Figure 8).

The IR spectrum of used EP solution is also shown in Figure 8. The predominant fluorosulfonic acid feature $\left(808 \mathrm{~cm}^{-1}\right)$ is reduced upon use of the EP solution, indicating that this peak can be tracked by IR to monitor the EP process. Again, no new peaks appear in the used EP spectrum to indicate EP product formation.

\section{NMR Spectra}

The presence of fluorosulfonic acid in EP solution is also confirmed with ${ }^{19} \mathrm{~F}$ NMR, and the spectra of new EP solution, new EP solution with added fluorosulfonic acid, and new EP solution with added water are shown in Figure 9. The NMR measurements allowed us to determine that new EP solution contains 1.61 mols of fluorine per $\mathrm{kg}$ of solution, of which 1.29 mols is bound in fluorosulfonic acid. Used EP solution, which contains $\sim 2 \mathrm{~g} / 1$ of dissolved niobium, also still contains 0.80 mols of fluorine bound in fluorosulfonic acid and only 0.01 mols of hydrofluoric acid per $\mathrm{kg}$ of solution. The used EP solution in this experiment does not represent completely exhausted solution. The peak near $-170 \mathrm{ppm}$, which is representative of the fluorine associated with hydrofluoric acid downshifts a little in the new EP solution diluted with water. This is representative of the difference between the local chemical environment provided by each solvent as well as the differing relative concentrations of $\mathrm{F}^{-}, \mathrm{FHF}^{-}$, and 
$\mathrm{HF}$ in each solution $[20,21]$. Although $\mathrm{F}^{-}, \mathrm{FHF}^{-}$, and $\mathrm{HF}$ each have a unique peak location, only one peak appears in hydrofluoric acid solutions due to rapid exchange between the ions, and the peak location indicates the relative concentrations of these ions. The peak location for various ratios of sulfuric acid/water/hydrofluoric acid is shown in Figure 10.

\section{DISCUSSION}

We determined the ratio of fluorine in hydrofluoric acid to fluorosulfonic acid, $\mathrm{R}$ $=[\mathrm{HF}] /\left[\mathrm{FSO}_{3}{ }^{-}\right]$, for various mixtures of hydrofluoric acid, sulfuric acid, fluorosulfonic acid, and water, and present the results in Table 4. We represent fluorosulfonic acid as the fluorosulfate ion, because fluorosulfonic acid is the strongest acid of the component species in the mixtures and the signature vibration of this ion was present in the Raman and IR spectra of new EP solution. Since hydrofluoric acid is a weaker acid, we represent it as HF, although its NMR signature contains contributions from HF, FHF', and $\mathrm{F}^{-}$. We did not actually determine the ionization extent of either acid, and only intend to represent the balance between the fluorine related to each acid with R. We determined $\mathrm{R}$ to be 0.25 for new EP solution. So, initially there is a small amount of fluorine available for dissolving the niobium, and as it is used (5) runs in reverse, slowly supplying more fluorine. The equilibrium for this reaction has previously been briefly discussed in terms of niobium electropolishing by Saito et al. [2], who suggested adding fluorosulfonic acid and water to used EP solution to refresh it. The NMR results that we present in Table 4 show that water can simply be added to the EP solution to force the remaining fluorosulfonic acid to supply additional fluorine. 
Via conductivity measurements, Gillespie et al. [22] determined that the equilibrium constant for (5) decreases with increasing temperature. Increasing the temperature also decreases the viscosity of the solution, therefore the free fluorine available for polishing is increased, and subsequently the etch rate, by two mechanisms. The addition of water also increases the amount of fluorine available for etching by the same two mechanisms. Our NMR data presented in Table 4 show that increasing the amount of water in the solution increases the $\mathrm{R}$, and the viscosities of sulfuric acid and water are $23 \mathrm{cp}$ and $0.9 \mathrm{cp}$, respectively, at $25^{\circ} \mathrm{C}$ [23]. Therefore, the etch rate would increase.

Not only do these results add to the knowledge of the chemistry of EP, they also suggest that EP could be controlled by a chemical component that binds the fluorine and slowly releases it. Thus we are provided with a route to explore potential new recipes for niobium EP with fluorinated components that are safer to handle and more effective than concentrated hydrofluoric acid.

Finally, we have shown that the amount of fluorine available for polishing from the standard EP recipe can be tracked by NMR or Raman (or possibly very roughly by IR) spectroscopy. Raman spectroscopy is more suitable than IR because the differences between new and used EP solution are more striking than they are in IR and there are two fluorosulfonic acid peaks available for calibration, whereas there is only one suitable 
peak available in the IR spectrum. NMR provides two suitable signals in the ${ }^{19} \mathrm{~F}$ spectrum for tracking.

\section{ACKNOWLDGEMENTS}

This work was supported in part by the U.S. Department of Energy under Contract No.

DE-AC02-07CH11359. We are grateful to D. N. Seidman for reading the manuscript. 
Table 1. Raman peaks in the EP solution and sulfuric acid (SA) spectra.

\begin{tabular}{|c|c|c|c|c|c|}
\hline \multicolumn{2}{|c|}{ New EP } & \multicolumn{2}{|c|}{1 vol. $96 \%$ SA : 0 vol. water } & \multicolumn{2}{|c|}{1 vol. $96 \%$ SA : 1 vol. water } \\
\hline $\begin{array}{c}\text { Location } \\
\left(\mathrm{cm}^{-1}\right)\end{array}$ & $\begin{array}{c}\text { Relative } \\
\text { Intensity (\%) }\end{array}$ & $\begin{array}{c}\text { Location } \\
\left(\mathrm{cm}^{-1}\right)\end{array}$ & $\begin{array}{c}\text { Relative } \\
\text { Intensity (\%) }\end{array}$ & $\begin{array}{c}\text { Location } \\
\left(\mathrm{cm}^{-1}\right)\end{array}$ & $\begin{array}{c}\text { Relative } \\
\text { Intensity (\%) }\end{array}$ \\
\hline 1390 & 4 & 1390 & 12 & 1210 & 18 \\
\hline 1166 & 28 & 1150 & 38 & 1050 & 100 \\
\hline 1082 & 65 & 1050 & 41 & 985 & 82 \\
\hline 1053 & 68 & 990 & 20 & 910 & 40 \\
\hline 915 & 100 & 915 & 100 & 600 & 47 \\
\hline 810 & 8 & 570 & 49 & 435 & 48 \\
\hline 565 & 65 & 415 & 40 & & \\
\hline 402 & 60 & & & & \\
\hline
\end{tabular}


Table 2. Raman and IR peaks of niobium-fluoride salts from [18].

\begin{tabular}{cccc}
\multicolumn{2}{c}{$\mathrm{K}_{2} \mathrm{NbOF}_{5}-\mathrm{H}_{2} \mathrm{O}($ Raman $)$} & \multicolumn{2}{c}{$\mathrm{CsNbF}_{6}($ Raman $)$} \\
\hline Location $\left(\mathrm{cm}^{-1}\right)$ & Intensity & Location $\left(\mathrm{cm}^{-1}\right)$ & Intensity \\
\hline 295 & medium & 280 & medium \\
600 & v. weak & 562 & weak \\
935 & medium & 683 & strong \\
\hline $\mathrm{K}_{2} \mathrm{NbOF}_{5}-\mathrm{H}_{2} \mathrm{O}\left(\mathrm{IR}^{-1} \mathrm{~cm}^{-1}\right)$ & $\mathrm{K}_{2} \mathrm{NbF}_{7}($ Raman $)$ \\
\hline 738 & $1626^{*}$ & 388 & medium \\
932 & $3572^{*}$ & 630 & v. strong \\
1083 & $3643^{*}$ & 782 & weak \\
\hline
\end{tabular}

*from water of hydration 
Table 3. IR peaks in new EP solution, sulfuric acid (SA), hydrofluoric acid (HFA), and water spectra.

\begin{tabular}{|c|c|c|c|c|c|}
\hline \multicolumn{2}{|c|}{ New EP } & \multicolumn{2}{|c|}{1 vol. $96 \%$ SA : 0 vol. water } & \multicolumn{2}{|c|}{1 vol. $96 \%$ SA : 1 vol. water } \\
\hline Location $\left(\mathrm{cm}^{-1}\right)$ & $\begin{array}{c}\text { Relative } \\
\text { Absorbance (\%) }\end{array}$ & Location $\left(\mathrm{cm}^{-1}\right)$ & $\begin{array}{c}\text { Relative } \\
\text { Absorbance }(\%)\end{array}$ & Location $\left(\mathrm{cm}^{-1}\right)$ & $\begin{array}{c}\text { Relative } \\
\text { Absorbance (\%) }\end{array}$ \\
\hline 2808 & 34 & 2849 & 28 & 3388 & 23 \\
\hline 2437 & 29 & 2411 & 20 & 2893 & 21 \\
\hline 2185 & 24 & 2162 & 12 & 2516 & 13 \\
\hline 1660 & 18 & 1654 & 5 & 2210 & 9 \\
\hline 1360 & 22 & 1348 & 31 & 1691 & 28 \\
\hline 1120 & 67 & 1127 & 57 & 1291 & 8 \\
\hline 1076 & 59 & 1053 & 31 & 1131 & 74 \\
\hline 1036 & 67 & 941 & 94 & 1018 & 100 \\
\hline 947 & 89 & 886 & 100 & 869 & 77 \\
\hline 887 & 100 & \multicolumn{2}{|c|}{1 vol. $49 \%$ HFA : 0 vol. water } & \multicolumn{2}{|c|}{1 vol. $49 \%$ HFA : 1 vol. water } \\
\hline 808 & 52 & 3484 & 100 & 3370 & 100 \\
\hline \multicolumn{2}{|c|}{ Water } & $\sim 3284$ & $\sim 78$ & $\sim 3300$ & $\sim 95$ \\
\hline 3295 & 100 & 2779 & 63 & $\sim 2783$ & $\sim 27$ \\
\hline 2114 & 7 & 2193 & 14 & 2179 & 1 \\
\hline \multirow[t]{4}{*}{1635} & 50 & 1755 & 91 & 1807 & 40 \\
\hline & & 1632 & 100 & 1630 & 84 \\
\hline & & $\sim 1160$ & 28 & 1175 & 5 \\
\hline & & 939 & 46 & 1026 & 8 \\
\hline
\end{tabular}


Table 4. The fluorine distribution in mixtures of $96 \%$ sulfuric acid (SA), $49 \%$ hydrofluoric acid (HFA), fluorosulfonic acid (FSA), and water determined from ${ }^{19} \mathrm{~F}$ NMR measurements.

\begin{tabular}{|c|c|c|c|c|}
\hline \multicolumn{4}{|c|}{ Initial Volume Ratios of Components in the Mixtures } & \multirow{2}{*}[\mathrm{HF}]{$/\left[\mathrm{FSO}_{3}^{-}\right]$} \\
\hline SA & HFA & FSA & Water & \\
\hline 0.900 & 0.100 & 0.000 & 0.000 & 0.25 \\
\hline 0.891 & 0.099 & 0.010 & 0.000 & 0.24 \\
\hline 0.855 & 0.095 & 0.050 & 0.000 & 0.21 \\
\hline 0.720 & 0.080 & 0.200 & 0.000 & 0.11 \\
\hline 0.720 & 0.080 & 0.000 & 0.200 & 6.26 \\
\hline
\end{tabular}




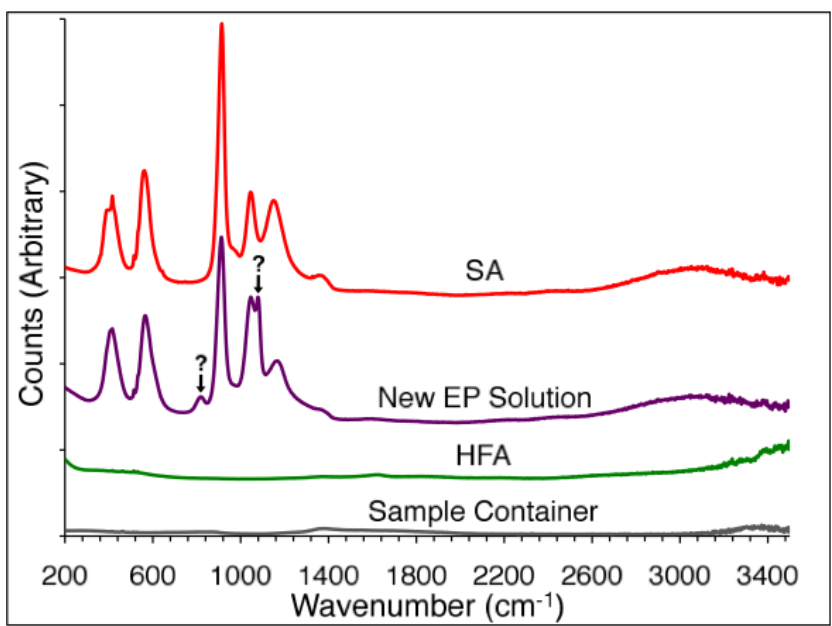

Figure 1. Raman spectra of new (unused) EP solution, its components (49\% hydrofluoric acid in water (HFA) and $96 \%$ sulfuric acid (SA)), and the sample container. The spectra are plotted with an offset for clarity. The question marks/arrows indicate peaks in the new EP solution spectrum that cannot be explained by the separate component spectra. 


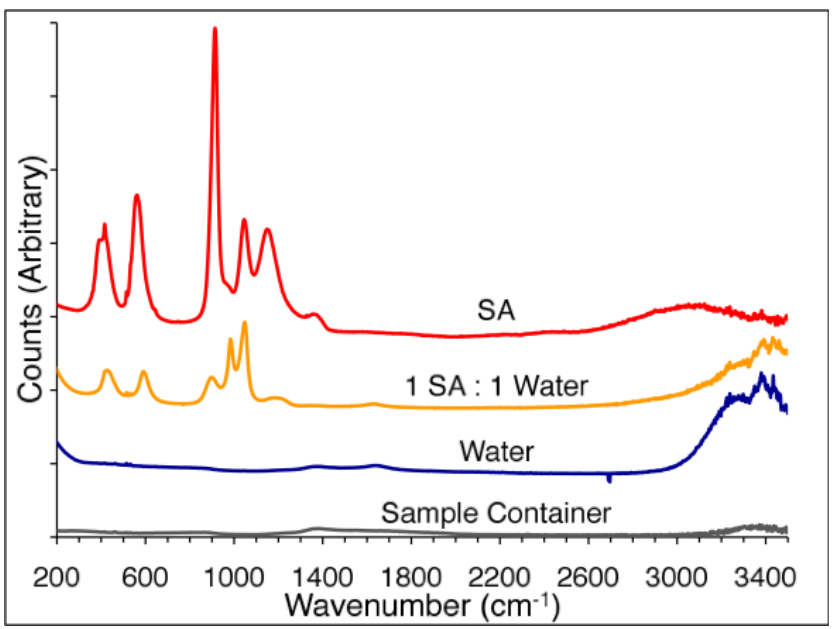

Figure 2. Raman spectra of 1 volume $96 \%$ sulfuric acid (SA) diluted with 1 volume water. The spectra of $96 \%$ sulfuric acid (component of EP solution), water, and the sample container are shown for reference. The spectra are plotted with an offset for clarity. 


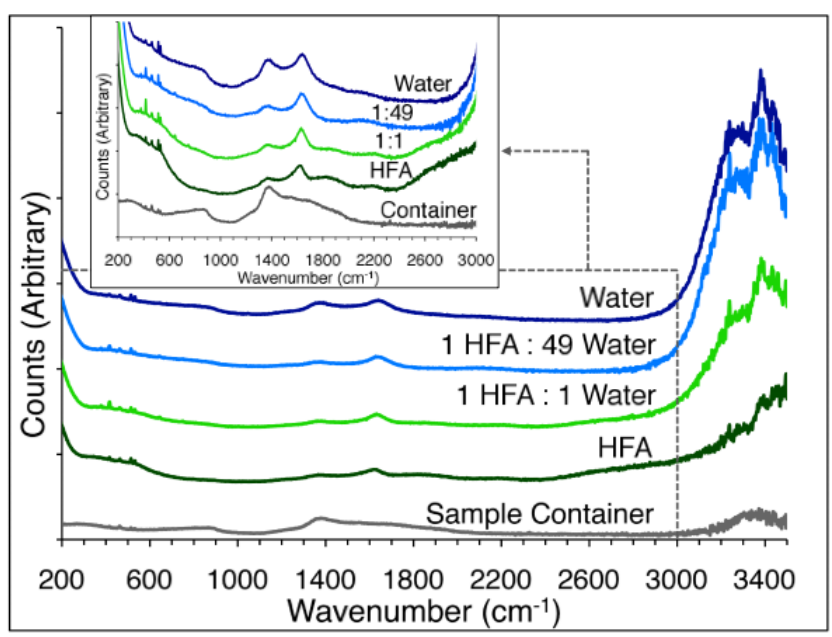

Figure 3. Raman spectra of $49 \%$ hydrofluoric acid (HFA) diluted with water; the ratios are by volume. The spectra of $49 \%$ hydrofluoric acid (component of EP solution), water, and the sample container are shown for reference. The spectra are plotted with an offset for clarity. The inset shows an expansion along the counts axis for the region 200-3000 $\mathrm{cm}^{-1}$. 


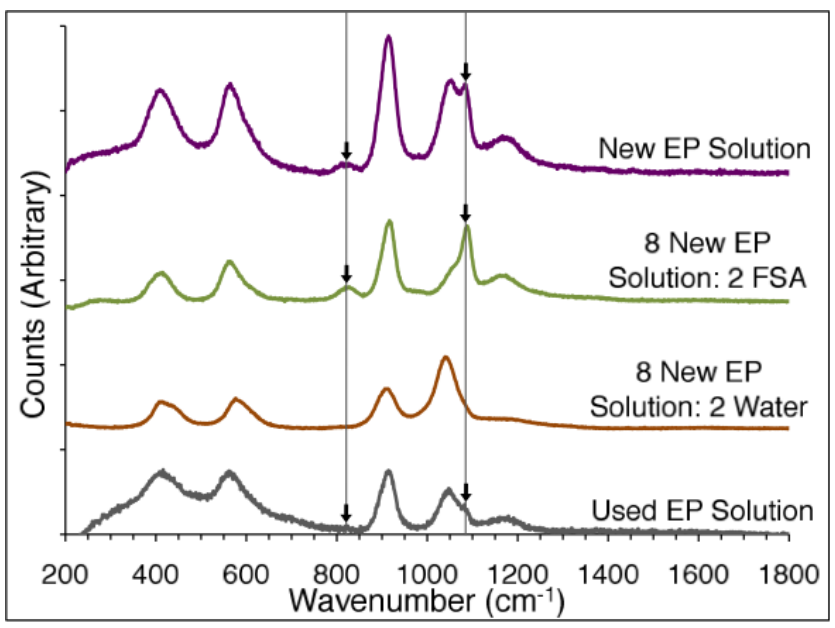

Figure 4. Raman spectra of new (unused) EP solution, 80 vol. \% EP solution +20 vol. \% fluorosulfonic acid (FSA), 80 vol. \% EP solution +20 vol. \% water, and used EP solution. The spectra are plotted with an offset for clarity. The arrows indicate peaks in the new and used EP solution spectra that are explained by the addition of fluorosulfonic acid. 


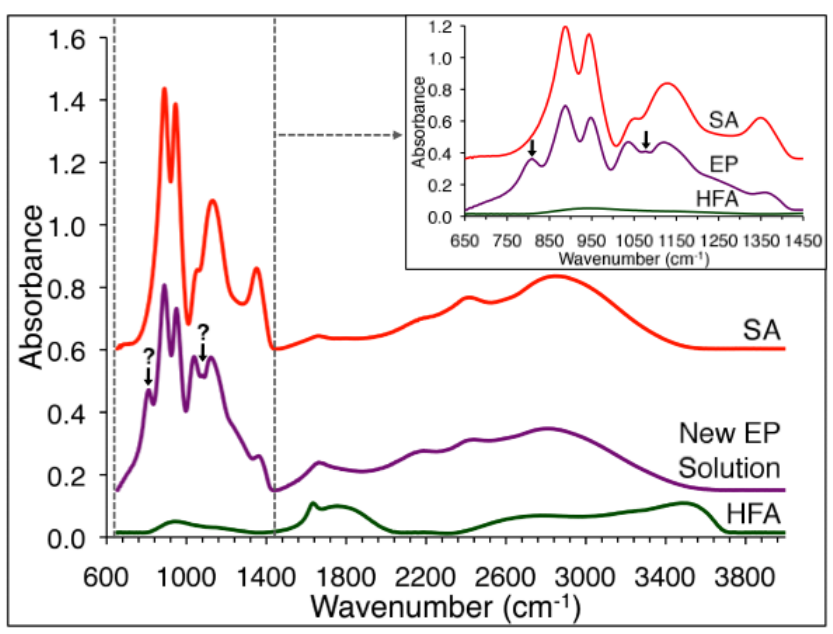

Figure 5. Infrared spectra of new (unused) EP solution and its components (49\% hydrofluoric acid (HFA) in water and $96 \%$ sulfuric acid (SA)). The spectra are plotted with an offset for clarity. The inset shows an expansion of the region $650-1450 \mathrm{~cm}^{-1}$ to emphasize the differences between new EP solution and its individual components. The question marks/arrows indicate peaks in the new EP solution spectrum that cannot be explained by the separate component spectra. 


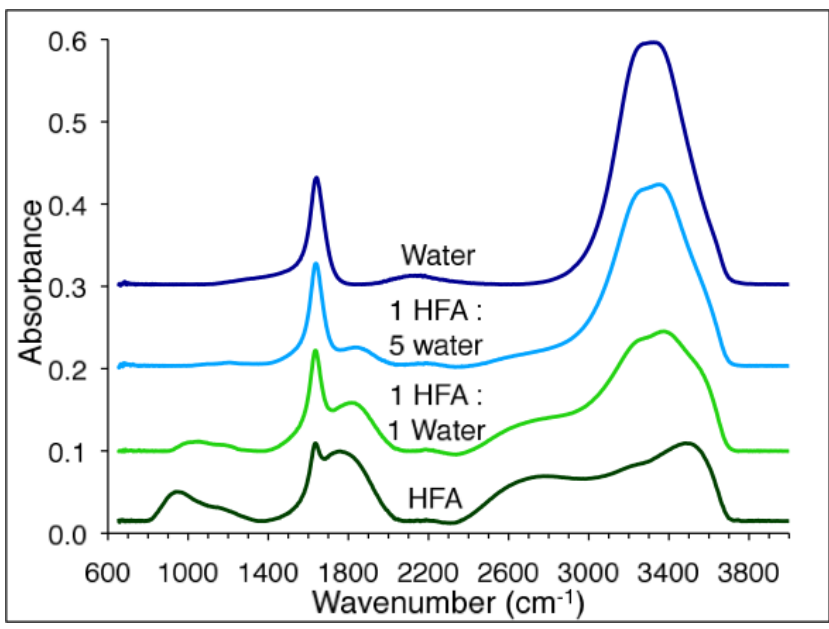

Figure 6. Infrared spectra of $49 \%$ hydrofluoric acid (HFA) diluted with water; the ratios are by volume. The spectra of $49 \%$ hydrofluoric acid (component of EP solution), and water are shown for reference. The spectra are plotted with an offset for clarity. 


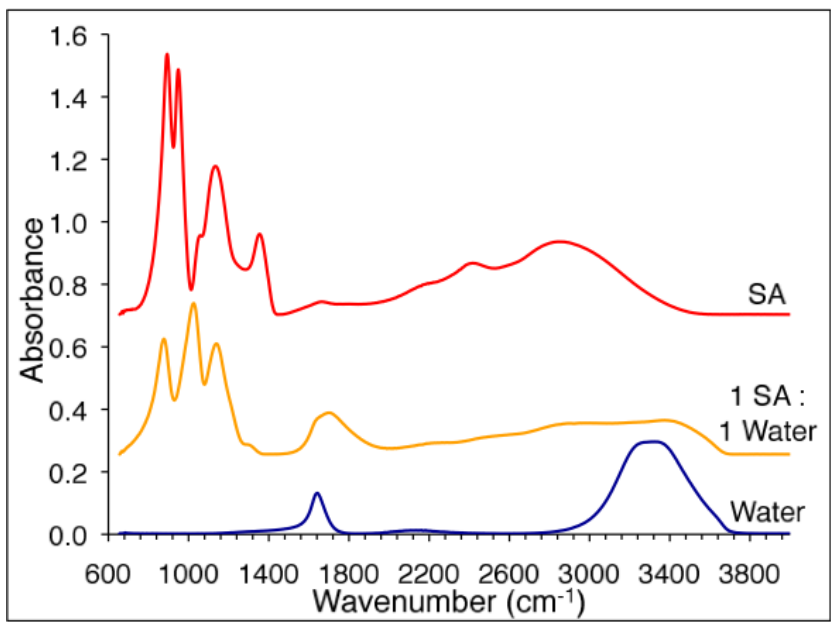

Figure 7. Infrared spectra of 1 volume 96\% sulfuric acid (SA) diluted with 1 volume water. The spectra of $96 \%$ sulfuric acid (component of EP solution) and water are shown for reference. The spectra are plotted with an offset for clarity. 


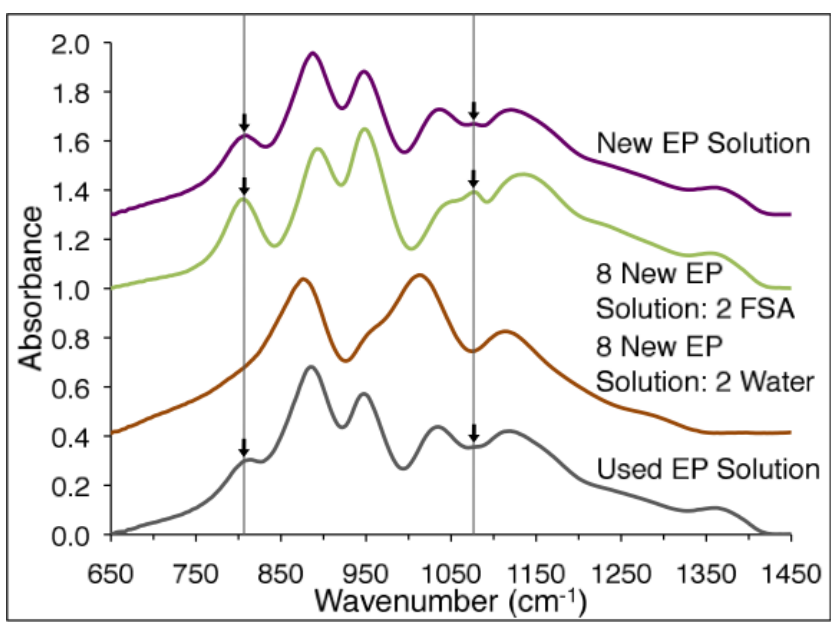

Figure 8. Infrared spectra of new (unused) EP solution, 80 vol. $\%$ EP solution +20 vol. $\%$ fluorosulfonic acid (FSA), 80 vol. \% EP solution +20 vol. \% water, and used EP solution. The spectra are plotted with an offset for clarity. The arrows indicate peaks in the new and used EP solution spectra that are explained by the addition of fluorosulfonic acid. 


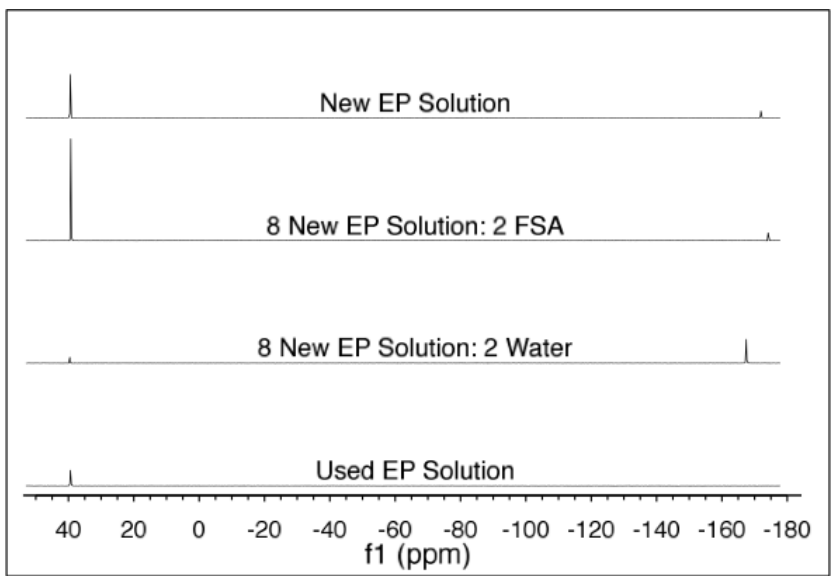

Figure 9. ${ }^{19} \mathrm{~F}$ NMR spectra of new (unused) EP solution, 80 vol. \% EP solution +20 vol. $\%$ fluorosulfonic acid (FSA), 80 vol. \% EP solution +20 vol. \% water, and used EP solution. The peak near $40 \mathrm{ppm}$ indicates fluorosulfonic acid and the peak near $-170 \mathrm{ppm}$ indicates hydrofluoric acid. 


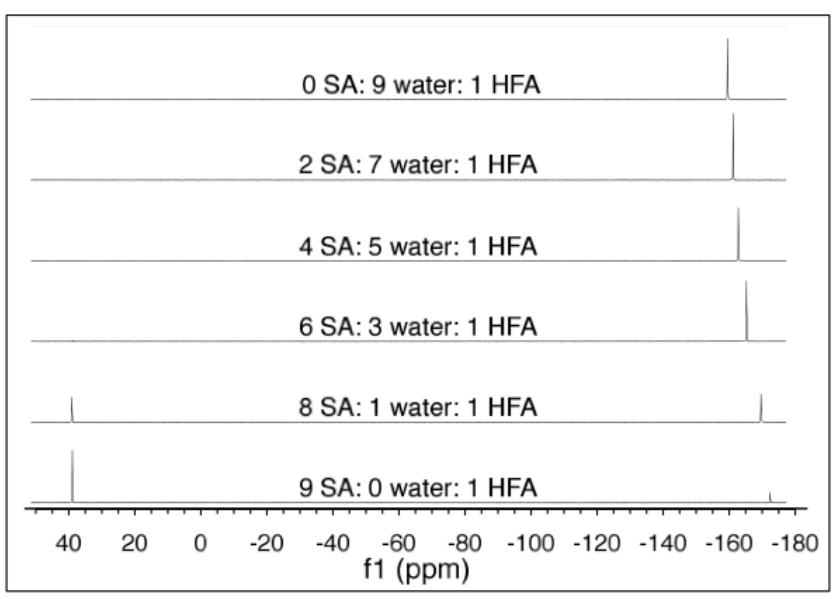

Figure 10. ${ }^{19} \mathrm{~F}$ NMR spectra of $48 \%$ hydrofluoric acid (HFA) diluted with various amounts of water and $95 \%$ sulfuric acid (SA); the ratios are by volume. The peak near $40 \mathrm{ppm}$ indicates fluorosulfonic acid and the peak near $-170 \mathrm{ppm}$ indicates hydrofluoric acid. 
Table Captions

Table 1. Raman peaks in the EP solution and sulfuric acid (SA) spectra.

Table 2. Raman and IR peaks of niobium-fluoride salts from [18].

Table 3. IR peaks in new EP solution, sulfuric acid (SA), hydrofluoric acid (HFA), and water spectra.

Table 4. The fluorine distribution in mixtures of $96 \%$ sulfuric acid (SA), $49 \%$ hydrofluoric acid (HFA), fluorosulfonic acid (FSA), and water determined from ${ }^{19} \mathrm{~F}$ NMR measurements.

\section{Figure Captions}

Figure 1. Raman spectra of new (unused) EP solution, its components (49\% hydrofluoric acid in water (HFA) and $96 \%$ sulfuric acid (SA)), and the sample container. The spectra are plotted with an offset for clarity. The question marks/arrows indicate peaks in the new EP solution spectrum that cannot be explained by the separate component spectra.

Figure 2. Raman spectra of 1 volume $96 \%$ sulfuric acid (SA) diluted with 1 volume water. The spectra of $96 \%$ sulfuric acid (component of EP solution), water, and the sample container are shown for reference. The spectra are plotted with an offset for clarity.

Figure 3. Raman spectra of $49 \%$ hydrofluoric acid (HFA) diluted with water; the ratios are by volume. The spectra of $49 \%$ hydrofluoric acid (component of EP solution), water, and the sample container are shown for reference. The spectra are plotted with an offset for clarity. The inset shows an expansion along the counts axis for the region 200-3000 $\mathrm{cm}^{-1}$.

Figure 4. Raman spectra of new (unused) EP solution, 80 vol. \% EP solution +20 vol. \% fluorosulfonic acid (FSA), 80 vol. \% EP solution +20 vol. \% water, and used EP solution. The spectra are plotted with an offset for clarity. The arrows indicate peaks in the new and used EP solution spectra that are explained by the addition of fluorosulfonic acid.

Figure 5. Infrared spectra of new (unused) EP solution and its components (49\% hydrofluoric acid (HFA) in water and $96 \%$ sulfuric acid (SA)). The spectra are plotted with an offset for clarity. The inset shows an expansion of the region $650-1450 \mathrm{~cm}^{-1}$ to emphasize the differences between new EP solution and its individual components. The question marks/arrows indicate peaks in the new EP solution spectrum that cannot be explained by the separate component spectra. 
Figure 6. Infrared spectra of $49 \%$ hydrofluoric acid (HFA) diluted with water; the ratios are by volume. The spectra of $49 \%$ hydrofluoric acid (component of EP solution), and water are shown for reference. The spectra are plotted with an offset for clarity.

Figure 7. Infrared spectra of 1 volume 96\% sulfuric acid (SA) diluted with 1 volume water. The spectra of $96 \%$ sulfuric acid (component of EP solution) and water are shown for reference. The spectra are plotted with an offset for clarity.

Figure 8. Infrared spectra of new (unused) EP solution, 80 vol. $\%$ EP solution +20 vol. $\%$ fluorosulfonic acid (FSA), 80 vol. \% EP solution +20 vol. \% water, and used EP solution. The spectra are plotted with an offset for clarity. The arrows indicate peaks in the new and used EP solution spectra that are explained by the addition of fluorosulfonic acid.

Figure 9. ${ }^{19} \mathrm{~F}$ NMR spectra of new (unused) EP solution, 80 vol. \% EP solution +20 vol. $\%$ fluorosulfonic acid (FSA), 80 vol. \% EP solution +20 vol. \% water, and used EP solution. The peak near $40 \mathrm{ppm}$ indicates fluorosulfonic acid and the peak near $-170 \mathrm{ppm}$ indicates hydrofluoric acid.

Figure 10. ${ }^{19} \mathrm{~F}$ NMR spectra of $48 \%$ hydrofluoric acid (HFA) diluted with various amounts of water and $95 \%$ sulfuric acid (SA); the ratios are by volume. The peak near $40 \mathrm{ppm}$ indicates fluorosulfonic acid and the peak near $-170 \mathrm{ppm}$ indicates hydrofluoric acid. 
References

[1] Diepers H, Schmidt O, Martens H and Sun F S 1971 New method of electropolishing niobium Phys. Lett. A A 37 139-40

[2] Saito K, Kojima Y, Furuya T, Mitsunobu S, Noguchi S, Hosoyama K, Nakazato T, Tajima T and Asano K 1989 In: Proceedings of the 4th Workshop on RF Superconductivity, (KEK, Tsukuba, Japan p 635-694)

[3] Casalbuoni S, Knabbe E-A, Kotzler J, Lilje L, von Sawilski L, Schmuser P and Steffen B 2005 Surface superconductivity in niobium for superconducting RF cavities Nucl. Instrum. Meth. A 538 45-64

[4] Saito K, Inoue H, Kako E, Fujino T, Noguchi S, Ono M and Shishido T 1998 Superiority of electropolishing over chemical polishing on high gradients Part Accel 60 193-217

[5] Zhao X, Geng R-L, Tyagi P V, Hayano H, Kato S, Nishiwaki M, Saeki T and Sawabe M 2010 Surface characterization of $\mathrm{Nb}$ samples electropolished with real superconducting rf accelerator cavities Phys. Rev. ST Accel. Beams 13 124702-1-7

[6] Tian H, Corcoran S G, Reece C E and Kelley M J 2008 The mechanism of electropolishing of niobium in hydrofluoric-sulfuric acid electrolyte $J$. Electrochem. Soc. 155 D563-8

[7] Lee S-J, Lai J-J and Lin Y-T 2005 Simulation of the formation mechanism of a viscous layer for the electropolishing process WIT Transactions on Engineering Sciences 48 203-13

[8] Subramanian S, Xue Q, Trabia M, Chen Y and Schill R J 2002 Modeling and optimization of the chemical etching process in niobium cavities. In: 2002 International congress on advances in nuclear power plants, (Hollywood, Florida)

[9] Ciovati G, Higo T, Kneisel P, Mammosser J, Matheisen A, Michelato P, Padamsee H, Reschke D, Rothgeb T, Saeki T, Saito K, Visentin B, Weise H and Oevermann C 2008 Final surface preparation for superconducting cavities - an attempt to describe an optimized procedure. (Hamburg, Germany: DESY)

[10] Tian H and Reece C E 2010 Evaluation of the diffusion coefficient of fluorine during the electropolishing of niobium Phys. Rev. ST Accel. Beams 13 083502-1-7

[11] Eozenou F, Aspart A, Antoine C and Malki B 2003 Electropolishing of niobium: best EP parameters. In: $C A R E$

[12] Cooley L D, Burk D, Cooper C, Dhanaraj N, Foley M, Ford D, Gould K, Hicks D, Novitski R, Romanenko A, Schuessler R, Thompson C and Wu G 2010 Impact of forming, welding, and electropolishing on pitting and the surface finish of SRF cavity niobium Ieee T. Appl. Supercon. 21 2609-2614

[13] Bruchon M, Visentin B and Eozenou F 2007 Electropolishing on single and multi-cell: Comsol modeling. In: 13th International Workshop on $R F$ Superconductivity, (Beijing, China)

[14] Kneisel P, Stoltz O and Halbritter J 1974 Surface preparation and measurement of niobium used in high-frequency cavities J. Appl. Phys. 45 2296-301 
[15] Aspart A and Antoine C Z 2004 Study of the chemical behavior of hydrofluoric, nitric and sulfuric acids mixtures applied to niobium polishing Appl. Surf. Sci. 227 17-29

[16] Kang G, Lee K, Park H, Lee J, Jung Y, Kim K, Son B and Park H 2010 Quantitative analysis of mixed hydrofluoric and nitric acids using Raman spectroscopy with partial least squares regression Talanta 81 1413-7

[17] Gillespie R J and Robinson E A 1961 The Raman spectra of sulphuric, deuterosulphuric, fluorosulphuric, chlorosulphuric, and methanesulphonic acids and their anions Can. J. Chem. 40 644-57

[18] Keller O L 1963 Identification of complex ions of niobium(V) in hydrofluoric acid solutions by Raman and infrared spectroscopy Inorg. Chem. 2 783-7

[19] Vonbarner J H, Christensen E, Bjerrum N J and Gilbert B 1991 Vibrationalspectra of niobium $(\mathrm{V})$ fluoro and oxo fluoro complexes formed in alkali-metal fluoride melts Inorg Chem 30 561-6

[20] Schaumburg K and Deverell C 1968 Fluorine-19 nuclear magnetic resonance chemical shift of hydrofluoric acid in $\mathrm{H} 2 \mathrm{O}$ and $\mathrm{D} 2 \mathrm{O}$ solutions J. Am. Chem. Soc. 90 2495-9

[21] Christe K O and Wilson W W 1990 Nuclear magnetic resonance spectrum of the fluoride anion J. Fluorine Chem. 46 339-42

[22] Gillespie R J, Milne J B and Senior J B 1966 Inorg. Chem. 5 1233-5

[23] Dorsey N E 2003 Viscosity of water, sulfuric acid, liquid carbon dioxide, and certain organic liquids. In: International Critical Tables: Knovel 